\title{
Trousseau's syndrome with arterial thrombosis and venous thromboembolism in a patient with ovarian cancer
}

\author{
C Rajapaksha ${ }^{a}$ S Rathnayaka ${ }^{b}$, S Hemapriya ${ }^{c}$
}

\begin{abstract}
Background: Trousseau syndrome (TS), first described in 1865 refers to coagulopathy associated with underlying malignancy. It is the second leading cause of death among cancer patients. Deep vein thrombosis and superficial thrombophlebitis are the common manifestations of TS while arterial thrombosis is seen rarely. We present a patient with TS presenting with concomitant arterial and venous thrombosis.
\end{abstract}

Case presentation: A 73 years old otherwise healthy lady presented with swelling of right lower limb with gangrenous toes. Investigations revealed right lower limb
Deep Vein Thrombosis (DVT) and arterial thrombosis with underlying ovarian malignancy. Her investigation findings excluded paradoxical embolism, vasculitis and other thrombotic conditions. Surgical resection of the tumor was done, but attempted inferior vena caval filter insertion failed. Postoperatively, she developed pulmonary embolism, which was managed with anticoagulation. Unfortunately she developed ascending gangrene of the right leg with fatal sepsis.

Conclusion: Concomitant arterial and venous thrombosis is a rare manifestation of TS. It is important to suspect underlying occult malignancy in patients presenting with arterial or venous thrombosis.

Key words: Trousseau syndrome, ovarian cancer, arterial thrombosis, DVT, pulmonary embolism

Sri Lanka Journal of Obstetrics and Gynaecology 2019; 41: 81-84

DOI: http://doi.org/10.4038/sljog.v41i3.7896

\section{Background}

Venous thromboembolism (VTE) is a major cause of morbidity and mortality among cancer patients. According to literature up to $20 \%$ of cancer patients develop thromboembolic events and about $20 \%$ of patients with thromboembolism have an underlying malignancy ${ }^{1}$. In 1865 , Trousseau described a syn- drome of unexpected thrombosis, chronic DIC with microangiopathy, verrucous endocarditis and arterial embolism in cancer patients. Today, the term 'Trousseau's syndrome' (TS) describes any coagulopathy associated with cancer ${ }^{2}$ and it is the second leading cause of death among cancer patients.

${ }^{a}$ Relief House Officer, Teaching Hospital Kandy, Sri Lanka.

${ }^{\mathrm{b}}$ Registrar in Obstetrics and Gynecology, Teaching Hospital Kandy, Sri Lanka.

c Consultant Obstetrician and Gynaecologist, Teaching Hospital Kandy, Sri Lanka.

Correspondence: CR, e-mail: <chiranthirajapaksha@gmail.com>

Received $12^{\text {th }}$ July 2019 and revised version accepted $4^{\text {th }}$ August 2019.

iD https://orcid.org/0000-0001-6805-2948

Competing interest: The authors report no conflict of interest

This is an open-access article distributed under the terms of the Creative Commons Attribution 4.0 International License, which permits unrestricted use, distribution and reproduction in any medium provided the original author and source are credited. 
We report a challenging case of an elderly lady with ovarian cancer whose initial presentation was recurrent deep vein thrombosis (DVT) and arterial thrombosis.

\section{Case presentation}

In May 2019 a 73 years old lady presented with painful right leg swelling with blackish discoloration of both $3^{\text {rd }}$ and $4^{\text {th }}$ toes for 3 days. She was a mother of 2 children and had reached menopause about 20 years ago.

She had a history of right leg DVT extending up to external iliac vein 8 months back. Investigations identified a pelvic mass. She was started on warfarin, but had defaulted clinic follow up after a few weeks and has not continued warfarin thereafter. She denied recent leg trauma or immobilization. There was no fever, shortness of breath (SOB) or hemoptysis.

On examination there was swelling of right leg up to thigh with gangrenous $3^{\text {rd }}$ and $4^{\text {th }}$ toes. The popliteal, dorsalis pedis and posterior tibial arterial pulsations were absent. She had no cardiac murmurs or lung signs. Abdominal examination revealed a hard fixed pelvic mass. Liver and spleen were not enlarged and there was no free fluid.

In view of her history of DVT, a recurrent DVT was suspected. However absent distal pulses with gangrene was consistent with concomitant arterial thrombosis or embolism. She was started empirically on intravenous heparin. Lower limb arterial doppler showed absent distal flow with possible obstruction at right profunda femoris artery. CT angiogram confirmed the presence of $100 \%$ obstruction at the same artery at mid-thigh level (Figure 1). Her basic hematology, renal and liver function tests were normal. Ultrasound of the pelvis showed a $9 \mathrm{~cm} \times 7 \mathrm{~cm} \times 6 \mathrm{~cm}$ mass with solid and cystic areas, papillary projections and increased vascularity, suggesting an ovarian carcinoma. Her CA-125 level was 70.9 (0-35), ESR was 60 mm/ $1^{\text {st }}$ hour, and Anti Nuclear antibody and anti cardiolipin antibody titers were negative.

A malignant pelvic mass causing pelvic vein compression resulting in DVT was diagnosed. However, concomitant arterial occlusion in this patient was unusual. Possible mechanisms considered were, hypercoagualability due to malignancy, local inflammation secondary to DVT predisposing adjacent arterial thrombosis, paradoxical embolism, vasculitis or other primary hypercoagulable states. Paradoxical embolism was excluded by a normal echocardiogram. Her investigations were also not in keeping with vasculitis or other conditions like myeloproliferative diseases, antiphospholipid antibody syndrome or paroxysmal nocturnal hemoglobinuria.

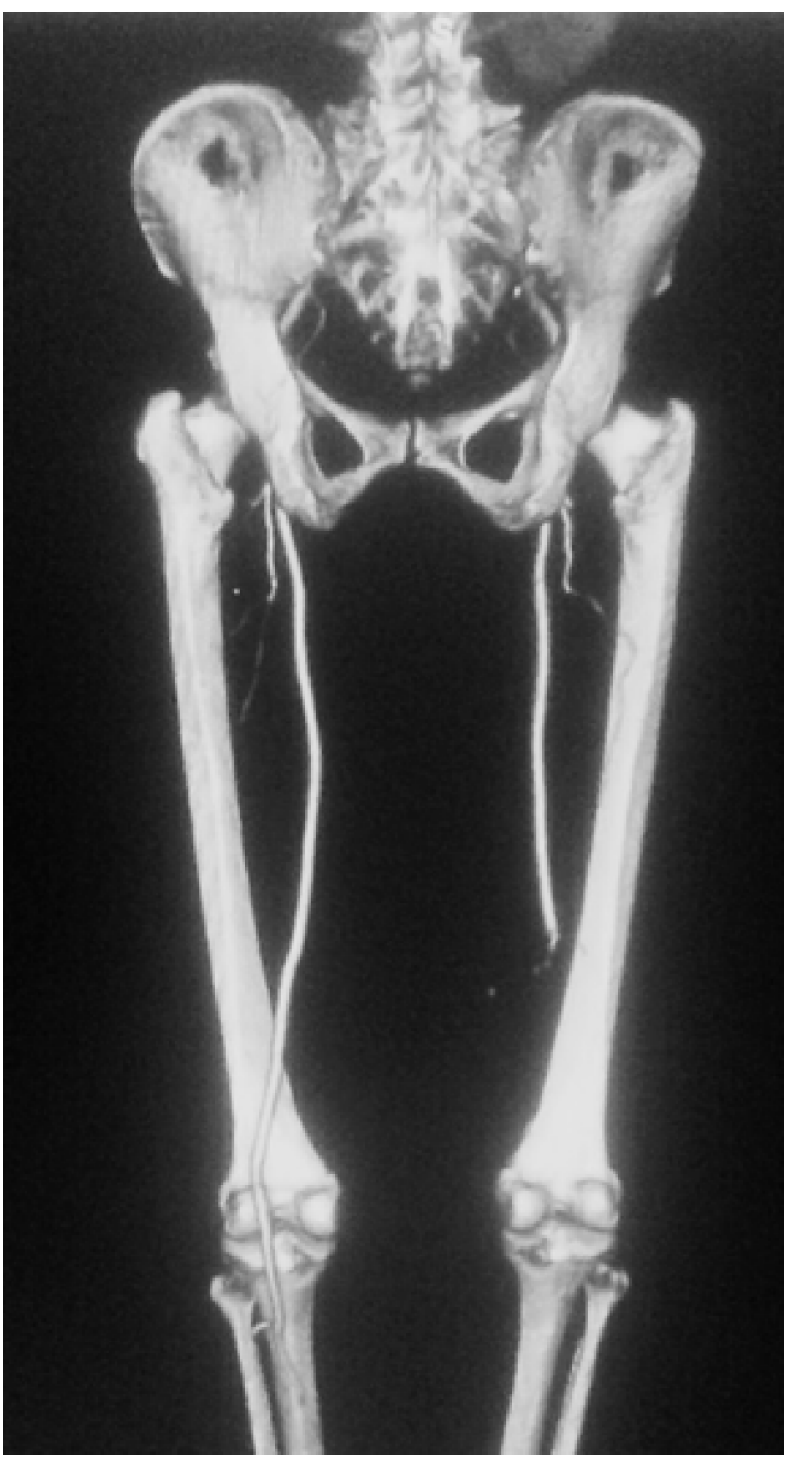

Figure. CT Angiogram of the patient - posterior view.

Following a multi-disciplinary meeting with contributions from an anaesthetist, gynaecologist, interventional radiologist and a vascular surgeon it was decided to initially insert an IVC filter to prevent pulmonary embolism (PE) and perform a laparotomy to stage and debulk the malignancy. Vascular bypass surgery was to be planned afterwards. Insertion of 
IVC filter was abandoned due to poor visualization. Intraoperatively a large adnexal mass was found infiltrating into pelvic walls with no metastasis. Total abdominal hysterectomy and bilateral salphingooophorectomy was performed. While on anticoagulation she developed acute SOB and desaturation on the second postoperative day, secondary to $\mathrm{PE}$, which was confirmed by a CTPA. Due to recent major surgery, it was decided not to thrombolyse. Anticoagulation and supportive management was continued. Tumor histology showed a high-grade serous micropapillary adenocarcinoma of ovary.

A few days later she developed ascending gangrene of right leg with sepsis. She was started on broadspectrum antibiotics and a foot amputation was offered. She was not in a state to give consent. Her next of kin did not give consent for amputation even after explaining the consequences. Despite aggressive management at the intensive care unit with broadspectrumantibiotics she passed away several days later.

\section{Discussion}

The term TS describes unexpected thrombosis that precedes the diagnosis of an occult visceral malignancy or appears concomitantly with the tumor ${ }^{2}$. Initially it was thought that mucin-producing tumors cause TS, but recently it was identified that tumors not producing mucin such as malignancies of pancreas (8.1\%), lung $(5.1 \%)$ and intestines $(4.9 \%)$ are also associated with the development of $\mathrm{TS}^{3}$.

Malignancy induced production of procoagulants and inhibition of synthesis of anticoagulants such as antithrombin III were the initially proposed mechanisms for TS. Later, bioactive agents produced by angiogenic epithelium causing hypercoagulability, such as tissue factor and cystine proteinases were discovered. In addition, malignancies increase platelet activation and cause endothelial dysfunction ${ }^{4}$. Cancer treatments including chemotherapy also add to the risk of thrombosis ${ }^{4}$.

Manifestations of TS include DVT and superficial thrombophlebitis. Arterial thrombosis is extremely rare and very few cases have been reported ${ }^{5}$. We were unable to find a patient who had all three DVT, PE and arterial thrombosis during the literature survey. Coronary artery disease and ischemic stroke have been described in association with malignancies but causal relationship is uncertain due to presence of comorbidities ${ }^{6}$.

Despite the advances in understanding the pathophysiology of TS, a diagnostic marker has not been identified. It is therefore largely a diagnosis of exclusion. We believe we were able to exclude most of the possible contributing factors for her presentation before diagnosing TS.

Definitive management for TS is removal of the causative tumor. Ideally an IVC filter should be inserted to prevent PE during and after surgery. Anticoagulation is recommended in all patients with TS. Conventional heparin is the drug with most benefit. In addition to its usual action, heparin interrupts carcinoma-mucin dependent adhesion phenomenon and neutralizes various cytokines and chemokines, further reducing thrombotic risk. Even though LMWHs are more convenient and effective, they do not confer those additional benefits 7 . There is no evidence for using vitamin $\mathrm{K}$ antagonists or novel oral anticoagulants in TS.

\section{Conclusion}

Concomitant arterial and venous thrombosis is rare in TS. It is important to suspect underlying occult malignancy in patients presenting with arterial or venous thrombosis. Further studies are required to identify diagnostic markers and set management guidelines for TS.

\section{Acknowledgements}

The authors wish to acknowledge the contributions from departments of haematology, radiology and vascular surgery of Teaching Hospital Kandy for the care of the patient.

\section{Authors' contributions}

All the authors contributed to the diagnosis and management of the patient. CR prepared the manuscript. All the authors reviewed and approved the final manuscript.

\section{References}

1. Metcalf RL, Fry DJ, Swindell R, McGurk A, Clamp AR, Jayson GC, Hasan J. Thrombosis in ovarian cancer: a case control study. Br J Cancer. 2014; 110: 1118-1124. doi: 10.1038/bjc.2014.3 
2. Varki A. Trousseau's syndrome: multiple definitions and multiple mechanisms. Blood. 2007; 110(6): 1723-1729. Doi: 10.1182/blood-2006-10-053736

3. Lyman GH. Venous thromboembolism in the patient with cancer. Cancer. 2010; 117(7), 13341349. Doi: $10.1002 /$ cncr.25714

4. Blann AD, Dunmore S. Arterial and Venous Thrombosis in Cancer Patients. Cardiology Research and Practice, 2011; 1-11. Doi: 10.4061/ 2011/394740

5. Prandoni P, Falanga A, Piccioli A. Cancer and venous thromboembolism. The Lancet Oncology, 2005; 6(6): 401-410. Doi: 10.1016/s1470-2045 (05) 70207-2

6. Silverberg D, Yalon T, Reinitz ER, Yakubovitch D, Segev T, Halak M. Acute limb ischemia in cancer patients: Aggressive treatment is justified. Vascular, 2015; 23(1): 55-61.

Doi: $10.1177 / 1708538114537048$
7. Grilz E, Königsbrügge $\mathrm{O}$, Posch F, et al. Frequency, risk factors, and impact on mortality of arterial thromboembolism in patients with cancer. Haematologica, 2018;103(9):1549-1556. Doi: 10.3324/haematol.2018.192419

\section{List of abbreviations}

DVT - Deep Vein Thrombosis

IVC - Inferior Vena Cava

TS - Trousseau Syndrome

VTE - Venous Thromboembolism

ESR - Erythrocyte Sedimentation Rate

ANA - Antinuclear Antibody

LMWH - Low Molecular Weight Heparin 